Ziemssen berichtete über eine an der Technischen Universität Dresden durchgeführte Studie, die Aufschluss über das Muster der autonomen Beteiligung bei Morbus Wilson liefern sollte. 26 Morbus Wilson-Patienten (zwölf davon mit neurologischem Phänotyp) und 26 gesunde Kontrollen wurden mit einem Fragebogen zu Symptomen des autonomen Nervensystems und mit Funktionstests im Hinblick auf eine autonome Dysfunktion untersucht, etwa mit HRV in Ruhe und während tiefer Respiration, Valsalva-Manöver, 24-Stunden-Herzfrequenz- und Blutdruckmessung oder BRS. Verwendet wurde eine trigonometrische regressive Spektralanalyse. Die Baroreflex-Messung zeigt die Interferenz zwischen Blutdruck und Herzfrequenz. 20 Patienten wurden nach drei Jahren erneut untersucht. Wie Ziemssen erläuterte, zeigten die Wilson-Patienten eine autonome Dysfunktion im Vergleich zur Kontrollgruppe vorwiegend als gesteigerte Herzfrequenz in Ruhe und im 24-Stunden-Mo- nitoring. Die BRS war in allen autonomen Funktionstests niedriger, insbesondere bei Patienten mit neurologischer Verlaufsform. Ein niedriger BRS gilt bei Patienten mit Parkinson, Postmyokardinfarkt oder Diabetes als Prognosefaktor für eine erhöhte Mortalität. Drei Jahre später hatte die BRS trotz Therapie weiter abgenommen. Insbesondere bei den neurologischen $\mathrm{Pa}-$ tienten war es zu einer parasympathischen Funktionsstörung gekommen. Wie Ziemssen zusammenfasste, war die kardiovaskuläre autonome Funktion bei gut behandeltem Morbus Wilson über drei Jahre grundsätzlich weitgehend stabil, die Abnahme des BRS müsse jedoch kritisch gesehen werden.

Dagmar Jäger-Becker, freie Medizinjournalistin

„Seltene neurologische Krankheiten: therapeutische Durchbrüche in

Sichtweite?" 89. DGN-Kongress, Mannheim, 21. - 24.9.2016

\title{
Einseitiger Sehverlust - was Neurologen wissen sollten
}

\author{
Plötzlicher Sehverlust eines Auges ist ein Problem, \\ vor dem auch Neurologen stehen können. Ein \\ Augenspiegel ist dann ein wesentliches diagnos- \\ tisches Requisit, zumal einige Störungen eine rasche \\ Intervention erforderlich machen.
}

Essenziell sind fünf Fragen als Basis einer raschen Orientierung zur Lokalisation, Pathophysiologie und Dringlichkeit eines akuten Visusverlusts, betonte Professor em. Detlef Kömpf, Universität Lübeck.

- Ist der Sehverlust monokulär? Dann sind entweder Auge oder Sehnerv vor dem Chiasma betroffen.

- Sieht das Auge normal aus oder finden sich bei Ausschluss von Carotissinus cavernosus-Fistel und endokriner Ophthalopathie eine Rötung, Schwellung oder ein Exophthalmus, die die Hinzuziehung eines Augenarztes erforderlich machen?

- Was ergibt die Abklärung eines „normalen“ Auges auf RAPD (rapid afferent pupillary defect) oder die Lichtreaktion mit dem Swinging-Flashlight-Test zur Differenzierung zwischen neuronalen und retinalen Problemen?

- Was ergibt die Funduskontrolle mit dem Augenspiegel?

- Ist die Augenmotilität gestört als Hinweis auf infra-/supranukleäre Paresen?

Neben gründlicher Anamnese vor allem hinsichtlich Beginn und Progression der Symptomatik sollte das kranielle Gefäßsystem abgehört beziehungsweise mit Doppler untersucht sowie eine kardiale Arrythmie abgeklärt werden. Mit dem Augenspiegel können Netzhautablösungen, retinale Ischämien und Papillenödem ebenso beurteilt werden wie eine Optikusatrophie und die Lokalisation des Sehnervs.

Exemplarisch für eine plötzliche reversible Sehstörung beschrieb Kömpf einen Patienten mit schmerzlosem „plötzlich herabfallendem Vorhang" im linken Auge, der sich rasch normalisierte. Er erwies sich als Amaurosis fugax mit lichtstarrer Pupille und - extrem selten nachweisbaren - kleinen reversiblen Cholesterinplaques im Gefäßsystem des Augenhintergrun- des. Die Amaurosis fugax ist zwar kein Notfall, erfordert aber rasch die Untersuchung auf atheromatöse Karotisplaques und kardiogene Emboliequellen und ist ein Indikator für Hirninfarkte. Bei persistierenden Sehstörungen sollte ein Astverschluss der Retinaarterie oder gar ein Zentralarterienverschluss abgeklärt werden, der als „kirschroter Fleck“ über Stunden bis Tage imponiert und danach in eine Optikusatrophie übergeht. Hier existiert allerdings keine wirksame Therapie, so der Neuroophthalmologe.

Eine rechtsseitig akut aufgetretene Sehstörung bei einem 70-jährigen Mann mit Hypertonie, Diabetes und dreifach Bypassoperation nach Herzinfarkt, bei der außer dem Visusverlust lediglich eine afferente Pupillenstörung nachweisbar war, stellte sich als akuter Papilleninfarkt (anteriore ischämische Optikusneuritis, AION) heraus. Ein solcher erfordert wegen häufigen Befalls auch des zweiten Auges eine konsequente Behandlung von Allgemein- und Risikofaktoren, so Kömpf.

Arteriitis temporalis lautete die Diagnose bei einem, seit Wochen unter allgemeinem Krankheitsgefühl, Gewichtsverlust und Kopfschmerzen leidenden 68-jährigen Mann mit Visusminderung des linken Auges und zunehmender Ptose sowie charakteristisch verhärteter, druckschmerzhafter Arteria temporalis. Eine solche Diagnose erfordert rasches Handeln noch vor dem Biopsieergebnis, um eine AION zu verhindern, mahnte Kömpf. Hier sei Prednison (1,0-1,5 mg/kg KG) über mindestens ein Jahr, ausschleichend unter BKS-Kontrolle notwendig, oder bei Erstmanifestation mit Visusverlust vorgeschaltet hochdosiertes Methylprednisolon (1 g/Tag i.v. über fünf Tage), um das häufig sukzessiv befallene zweite Auge zu schützen. Davon abzugrenzen sei die häufigere, nicht auf Steroide ansprechende, nichtarteriitische/idiopathische AION mit früherem Altersgipfel. Dies gelinge durch die Biopsie sowie durch die bei Arteriitis temporalis erhöhte $\mathrm{BSG}(>50 \mathrm{~mm} / \mathrm{h})$.

Dr. Andreas Häckel, freier Medizinjournalist

Vortrag Professor em. Dr. Detlef Kömpf, Lübeck: „Das vaskuläre Auge“;

89. DGN-Kongress, Mannheim, 21.-24.9.2016 